\title{
Short-interval Self-Learning to Improve Retention of Resuscitation Skills: A Randomized Controlled Trial
}

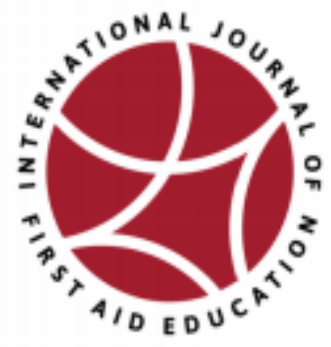

Ryuhei Sato, Chika Nishiyama, Kosuke Kiyohara, Makiko Sano, Tasuku Matsuyama, Tomonari Shimamoto, Toshihiro Hatakeyama, Taku Iwami

The optimal interval and methods for cardiopulmonary resuscitation (CPR) retraining remain unclear. We evaluated the difference in CPR skills one year after a 45-minute chest compression-only CPR training among participants receiving short self-learning refresher training at 3-month intervals, those receiving this training at 6-month intervals, and those without the refresher training.

Participants were randomly assigned to the 3-month group, 6-month group, or control group. Resuscitation skills were assessed immediately after the initial training, and at 1 year after the training. The primary endpoint was the number of chest compressions performed with appropriate depth one year after the training. Among 129 participants that were selected, 112 were fully evaluated. Although no significant differences were found in the baseline characteristics among the three groups, the self-training groups demonstrated a tendency to perform fewer chest compressions with appropriate depth $(p=.122)$. No significant differences were observed between the three groups in terms of the number of chest compressions with appropriate depth one year after the training $(p=.895)$. However, the number of appropriate chest compressions, the number of chest compressions with appropriate depth, and chest compression depth in the short-interval self-retraining groups one year later were better than those observed immediately after training.

Short self-retraining every 3 or 6 months after the chest compression-only CPR training could not be attributed to the retention of chest compression skills with appropriate depth. However, short, frequent self-retraining in persons with poor skills might be useful in acquiring chest compression skills.

Keywords: Cardiopulmonary resuscitation, Chest compression, Retraining

\section{Background}

Cardiopulmonary resuscitation (CPR) training courses are conducted worldwide to increase the proportion of bystander CPR. However, studies evaluating the retention of CPR performance have demonstrated rapid deterioration in CPR skills within 3-6 months after initial training (Andresen et al., 2008; Smith, Gilcreast, \& Pierce, 2008). Thus, regular repetition of hands-on training within short time intervals is vital to maintaining CPR skills (Niles et al., 2017; Oermann, Kardong-Edgren, \& Odom-Maryon, 2011). However, there are obstacles in attending traditional CPR training, including issues related to the time and cost of training (Wik, Brennan, \& Braslow, 1995). Due to limited time and resources, a short refresher training could be more acceptable to instructors and participants. Furthermore, a previous study examined the effectiveness of very short self-retraining programs with automated assessment and feedback at 3 months in a simulated randomized controlled trial (Nishiyama et al., 2017); however, long-term retention of CPR skills for short 
self-retraining remains unclear. According to the 2015 International Consensus on Cardiopulmonary Resuscitation and Emergency Cardiovascular Care Science with Treatment Recommendations (CoSTR), there is insufficient evidence regarding the optimal interval and method for long-term retraining of CPR skills (Bhanji et al., 2015).

Hence, this study aimed to evaluate the difference in retention of CPR skills 1-year after a 45-minute chest compression-only CPR training program among participants receiving short self-learning refresher training at 3-month and 6-month intervals, as well as those without self-learning refresher training.

\section{Methods}

\section{Study Design}

This study was conducted between February 2016 and February 2017 as a randomized controlled simulation study (ID: UMIN000020425).

\section{Ethical Considerations}

We obtained written informed consent from each participant, and the study was approved by the Ethics Committee of the Kyoto University Graduate School of Medicine (registration number: C1140). In compliance with ethical requirements, the trainees were not prohibited from participating voluntarily in CPR training aside from the self-learning refresher training conducted in the present study during the 1-year period.

\section{Recruitment and Randomization}

Students of Kyoto University aged $\geq 18$ years were invited to participate in this study through flyers as well as by word of mouth from participants themselves. As CPR training within six months is assumed to affect acquisition of CPR skills (Nyman \& Sihvonen, 2000), only students who had not attended CPR training within the preceding six months were included in this study. Participants with heart or orthopedic diseases were excluded. Participants were randomly assigned to one of three groups: those who were to undergo short self-learning refresher training at shorter intervals on a quarterly basis (3-month group), those who were to undergo this training at 6-month intervals (6-month group), and those who would not undergo any selflearning refresher training (control group). This randomization was performed using permuted blocks with an allocation ratio of 1:1:1 and stratified by sex, since sex may affect the quality of chest compressions (Ashton, McCluskey, Gwinnutt, \& Keenan, 2002). An independent biostatistician provided a computergenerated randomization list. All participants and instructors were aware of their allocations after their skills evaluation following the chest compression-only CPR training.

\section{Compression-Only CPR Training}

All participants underwent a 45-minute chest compression-only CPR training, which consisted of chest compression and automated external defibrillator (AED) use. The effectiveness of this training program has been previously reported (Nishiyama et al., 2014). The course used a digital video disc (DVD) and a very small personal resuscitation manikin (Mr.PUSH LIGHT $^{\circledR}$; Alexon Co., Ltd., Itami, Japan). Mr.PUSH LIGHT $^{\circledR}$ was designed for chest compression training and makes a sound when a trainee provides a compression depth of at least $50 \mathrm{~mm}$.

\section{Intervention}

The self-learning refresher training groups attended the sessions every three or six months after the chest compression-only CPR training. For participants in both self-learning refresher training groups, the practice of chest compression was conducted using Mr.PUSH PRO CPR501 (Alexon Co., Ltd., Itami, Japan), while the control group was exposed to neither self-learning training nor this device. The details of the device have been previously described (Nishiyama et al., 2017). In brief, the device, which is a model of the human thorax, has a sensor for detecting and offering real-time visual and audio feedback for chest compression depths, recoils, and compression rates during the training. The participants were asked to practice chest compressions using the device for one minute. To be deemed competent according to the Japanese CPR guidelines (Japan Resuscitation Council, 2016), all participants had to fulfill the following conditions: $\geq 90 \%$ compressions 
with a depth of $\geq 45 \mathrm{~mm}, \leq 10 \%$ compressions with incomplete release, and a compression rate $\geq 100 / \mathrm{min}$. Participants who were unable to fulfill the proficiency criteria were asked to continue practicing until they succeeded. The self-learning refresher training lasted for a maximum of $5 \mathrm{~min}$. The site coordinator assured whether the study protocol was followed or not but did not provide participants with any feedback during the self-training sessions.

\section{Outcome Measures}

We evaluated the participants' resuscitation skills using a full-sized Resusci Anne ${ }^{\circledR}$ SkillReporter ${ }^{\mathrm{TM}}$ and the Laerdal ${ }^{\circledR} \quad$ PC Skill Reporting ${ }^{\mathrm{TM}}$ software (Laerdal Medical, Stavanger, Norway) in a case-based scenario immediately and 1 year after the initial training. Each participant was individually called into the test room and presented with the following scenario: "Imagine that you are at a department store. Suddenly, a man collapses in front of you. You are the only person around. Do whatever you can to help this man."

The primary endpoint was the number of chest compressions with appropriate depth $(\geq 45 \mathrm{~mm})$ performed during a 2-min test period 1 year after the chest compression-only CPR training. The reason for selecting this primary endpoint was that while it is an essential factor affecting survival rate, it was difficult for the general public to acquire and maintain chest compression skills with appropriate depth among CPR techniques (Gyllenborg, Granfeldt, Lippert, Riddervold, \& Folke, 2017; Oermann et al., 2011).

The secondary endpoints were as follows: (a) the number of total chest compressions, (b) the number of appropriate chest compressions, (c) the number of chest compressions with complete release, (d) chest compression depth $(\geq 45 \mathrm{~mm})$, (e) compression rate (100-120 $\left.\mathrm{min}^{-1}\right)$, (f) period from the start of the presentation of scenario to the first chest compression, (g) time without chest compressions during the 2-min test period, (h) the initial assessment by the participant, (i) call for an ambulance (119), (j) call for AED, and (k) check for breathing. An appropriate chest compression was defined as one with adequate depth $(\geq 45 \mathrm{~mm})$, correct hand position, and complete recoil $(\leq 10 \mathrm{~mm})$ according to the Japanese CPR guidelines. The initial assessment by the participant, calls for an ambulance and AED, and check for breathing were evaluated by the instructors using a checklist.

\section{Statistical Analysis}

We estimated that the average number of chest compressions with appropriate depth during the 2-min test period 1 year after the initial training would be 140 times in the 3-month group, 120 times in the 6-month group, and 90 times in the control group based on previous data (Nishiyama et al., 2015; Sutton et al., 2011). With an alpha level of $5 \%$ and a power of $80 \%$, 39 participants were needed per group. Considering a possible $5 \%$ post-randomization dropout rate, a total sample size of 126 participants was used. Participants who were absent from the chest compression-only CPR training, lost to follow-up, or had no recorded data because of technical errors were not included in the analyses. The data were compared among the three groups using the chi-square test or Fisher's exact test for categorical variables and Kruskal-Wallis test for continuous variables. After logarithmic transformation of the chest compression data, a generalized linear model (GLM) analysis was used to compare the chest compression skills among the three groups. Using the control group as a reference, we calculated the coefficient with adjustments for age, sex, previous CPR training, and each dependent variable immediately after the chest compression-only CPR training. Differences in chest compression data within groups obtained immediately following the chest compression-only CPR training and 1 year after the training were tested using the Wilcoxon signed-rank test. Data were expressed as numbers with percentages, medians with interquartile ranges, or coefficients with $95 \%$ confidence intervals (CIs). All analyses were conducted using SPSS 24 (SPSS Inc., Chicago, IL, USA). $P$ values $<.05$ were considered significant.

\section{Results}

Participants were recruited in January 2016 and followed up until February 2017. This trial ended when the data analysis was completed. Among 129 enrolled 


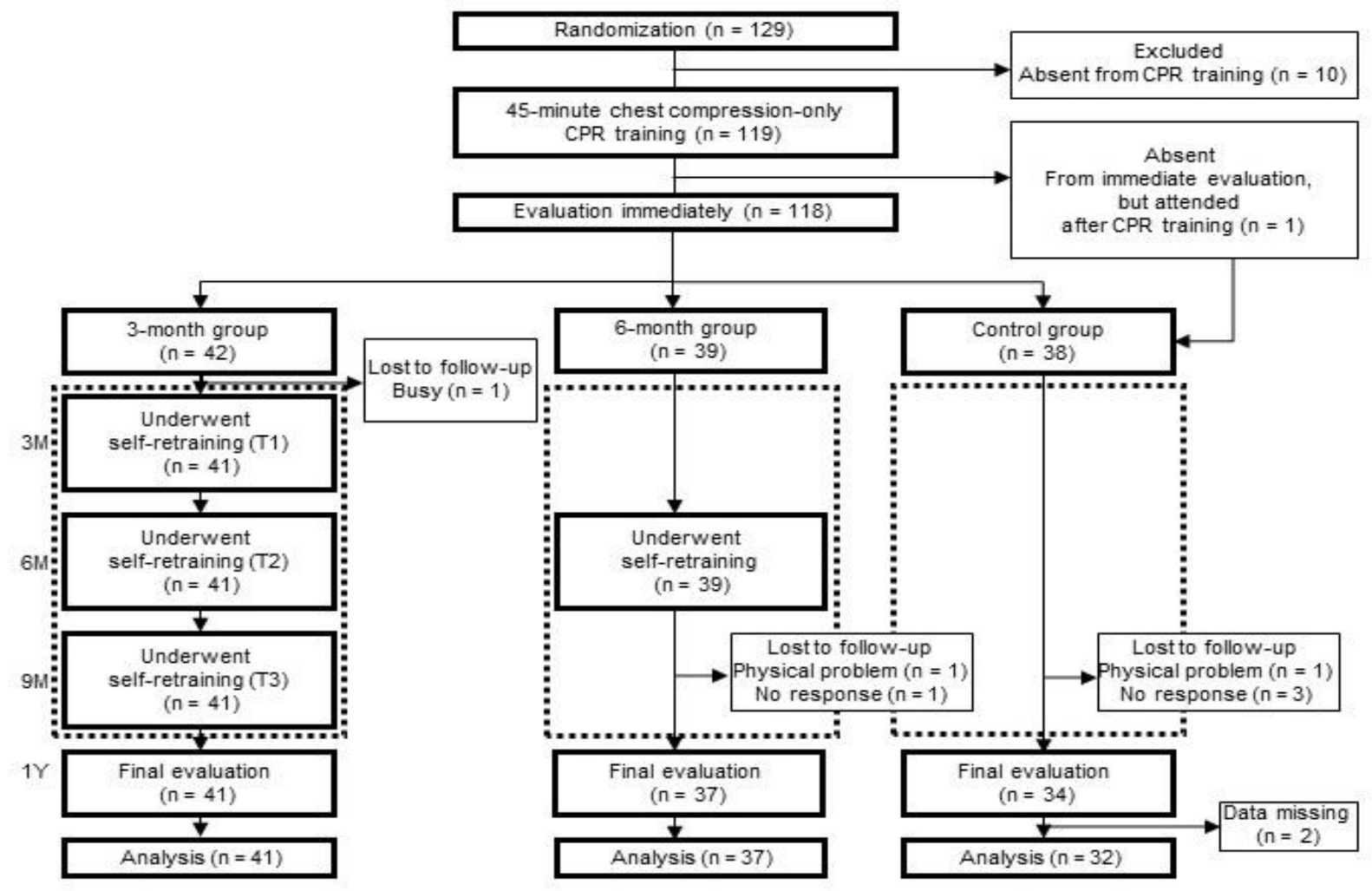

Figure 1. Flowchart for selection of study participants.

Table 1.

Characteristics of Participants and Resuscitation Skills Immediately after the Chest Compression-Only Cardiopulmonary Resuscitation Training

\begin{tabular}{|c|c|c|c|c|}
\hline & $\begin{array}{c}\text { 3-month group } \\
(n=41)\end{array}$ & $\begin{array}{c}\text { 6-month group } \\
(n=37)\end{array}$ & $\begin{array}{c}\text { Control group } \\
(n=32)\end{array}$ & $p$ \\
\hline Male, n (\%) & $6(14.6)$ & $3(8.1)$ & $4(12.5)$ & .706 \\
\hline Age, median (IQR), years & $21(20-21)$ & $20(20-21)$ & $21(20-21)$ & .789 \\
\hline Prior cardiopulmonary resuscitation training, $\mathrm{n}(\%)$ & $30(73.2)$ & $33(89.2)$ & $27(84.4)$ & .169 \\
\hline Witnessed sudden cardiac arrest, $\mathrm{n}(\%)$ & $5(12.2)$ & $5(13.5)$ & $6(18.8)$ & .716 \\
\hline Family history of sudden cardiac arrest, $n(\%)$ & $1(2.4)$ & $1(2.7)$ & $2(6.3)$ & .680 \\
\hline \multicolumn{5}{|l|}{ Recognition and activation of EMS, $\mathbf{n}(\%)^{a}$} \\
\hline Check for conscious & $41(100.0)$ & $37(100.0)$ & $29(93.5)$ & .079 \\
\hline Called for help (119) & $41(100.0)$ & $37(100.0)$ & $31(100.0)$ & 1.000 \\
\hline Called AED & $41(100.0)$ & $37(100.0)$ & $31(100.0)$ & 1.000 \\
\hline Check for breathing & $14(34.1)$ & $15(40.5)$ & $13(41.9)$ & .760 \\
\hline \multicolumn{5}{|l|}{ Chest compressions, $n$, median $(\mathrm{IQR})^{\mathrm{s}}$} \\
\hline Total number of chest compressions & $225(210-244)$ & $236(222-244)$ & $231(218-242)$ & .626 \\
\hline Appropriate chest compressions & $29(0-90)$ & $6(1-53)$ & $34(1-158)$ & .340 \\
\hline Chest compressions with appropriate depth & $40(1-96)$ & $26(1-94)$ & $104(5-191)$ & .122 \\
\hline Chest compressions with correct recoil & $225(202-242)$ & $229(214-242)$ & $230(213-242)$ & .819 \\
\hline Compression depth $[\mathrm{mm}]$ & $39(33-44)$ & $38(35-42)$ & $45(33-51)$ & .352 \\
\hline Compression rate $\left[\mathrm{min}^{-1}\right]$ & $113(105-122)$ & $118(110-122)$ & $115(108-120)$ & .681 \\
\hline \multicolumn{5}{|l|}{ Resuscitation time course, second, median (IQR) } \\
\hline Time to chest compression ${ }^{c}$ & $17(15-19)$ & $15(13-19)$ & $17(14-19)$ & .454 \\
\hline Time without chest compression during a 2-min test period & $0(0-0)$ & $0(0-0)$ & $0(0-0)$ & .412 \\
\hline
\end{tabular}
Note.

$\mathrm{IQR}=$ interquartile range; EMS = emergency medical services; $\mathrm{AED}=$ automated external defibrillator .

a3-month group $(n=41), 6$-month group $(n=37)$, Control group $(n=31)$

3-month group ( $n=40), 6$-month group $(n=35)$, Control group $(n=31)$

c3-month group $(n=40), 6$-month group $(n=37)$, Control group $(n=31)$

participants, 119 participated in the chest compressiononly CPR training. They were divided into 3-month, 6- month, and control groups with 42, 39, and 38 participants, respectively. One year later, the CPR skills 
of $41(97.6 \%)$ participants in the 3-month group, 37 $(94.9 \%)$ in the 6-month group, and $34(89.5 \%)$ in the control group were fully evaluated (Figure 1). Among these, two participants from the control group were excluded from the analysis as their data cannot be extracted because the computer froze and then crashed.

\section{Baseline Characteristics and Resuscitation Skills}

Table 1 presents the baseline characteristics and resuscitation skills of participants that were included in the analysis. Although the control group demonstrated a tendency for a high number of chest compressions with appropriate depth, there were no significant differences in the baseline characteristics among the three groups.

\section{Resuscitation Skills 1 Year Post}

No significant differences in the participants' CPR performance one year after the training were observed among the three groups, as presented in Table 2. With regard to the primary outcome (the number of chest compressions with appropriate depth), no significant differences were observed between the 3-month (85 [13-208]), 6-month (133 [21-212]), and control (93 [20-196]) groups $(p=.895)$. Results of GLM analysis showed that there were no significant differences in the number of chest compressions with appropriate depth $(\geq 45 \mathrm{~mm}$ ) in the 3-month (coefficient, 1.21; 95\% CI, $0.58-2.53$ ) and 6-month (coefficient, 1.32; 95\% CI, 0.62-2.81) groups compared with the control group. However, the coefficient for the total number of chest compressions (one year after the chest compressiononly CPR training) comparing the 3-month $(1.05$; $95 \%$ CI, 1.00-1.11) and 6-month (1.05; 95\% CI, 1.00-1.11) groups with the control group demonstrated significant differences.

A few participants arbitrarily participated in CPR training aside from the self-learning refresher training in the present study during the 1-year period. No significant differences were observed among the 3month (6 [14.6\%]), 6-month (4 [10.8\%]), and control (7 [21.9\%]) groups $(p=.440)$ (data not shown). In addition, none of the participants continued the selflearning refresher training for a maximum of $5 \mathrm{~min}$.

\section{Changes in Chest Compression Skills}

The changes in chest compression skills within the groups 1 year after the training are illustrated in Figure 2. The number of appropriate chest compressions at 1 year after the training was significantly greater than that observed immediately after the training with the 3-

Table 2 .

Resuscitation Skills 1 Year after the Chest Compression-Only Cardiopulmonary Resuscitation Training

\begin{tabular}{|c|c|c|c|c|}
\hline & $\begin{array}{c}\text { 3-month group } \\
(n=41)\end{array}$ & $\begin{array}{c}\text { 6-month group } \\
(n=37)\end{array}$ & $\begin{array}{c}\text { Control group } \\
(n=32)\end{array}$ & $p$ \\
\hline \multicolumn{5}{|l|}{ Recognition and activation of EMS, n (\%) } \\
\hline Check for conscious & $40(97.6)$ & $35(94.6)$ & $30(93.8)$ & .734 \\
\hline Called for help (119) & $32(78.0)$ & $30(81.1)$ & $27(84.4)$ & .792 \\
\hline Called AED & $35(85.4)$ & $33(89.2)$ & $32(100)$ & .065 \\
\hline Check for breathing & $18(43.9)$ & $14(37.8)$ & $19(59.4)$ & .186 \\
\hline \multicolumn{5}{|l|}{ Chest compressions, $\mathrm{n}$, median (IQR) } \\
\hline Total number of chest compressions & $221(210-235)$ & $224(210-239)$ & $211(192-232)$ & .059 \\
\hline Appropriate chest compressions & $58(8-175)$ & $63(3-208)$ & $72(8-184)$ & .982 \\
\hline Chest compressions with appropriate depth & $85(13-208)$ & $133(21-212)$ & $93(20-196)$ & .895 \\
\hline Chest compressions with correct recoil & $221(210-235)$ & $224(210-239)$ & $211(192-231)$ & .063 \\
\hline Compression depth $[\mathrm{mm}]$ & $44(38-53)$ & $46(39-51)$ & $45(39-52)$ & .949 \\
\hline Compression rate $\left[\mathrm{min}^{-1}\right]$ & $110(105-116)$ & $112(105-119)$ & $106(101-118)$ & .275 \\
\hline \multicolumn{5}{|l|}{ Resuscitation time course, second, median (IQR) } \\
\hline Time to chest compression ${ }^{\mathrm{a}}$ & $18(14-23)$ & $16(12-19)$ & $19(14-21)$ & .157 \\
\hline Time without chest compression during a 2 -min test period & $0(0-0)$ & $0(0-0)$ & $0(0-3)$ & .097 \\
\hline
\end{tabular}

EMS = emergency medical services; $\mathrm{AED}=$ automated external defibrillator; $I Q R=$ interquartile range

a-month group $(n=40)$, 6-month group $(n=36)$, Control group $(n=31)$ 
A

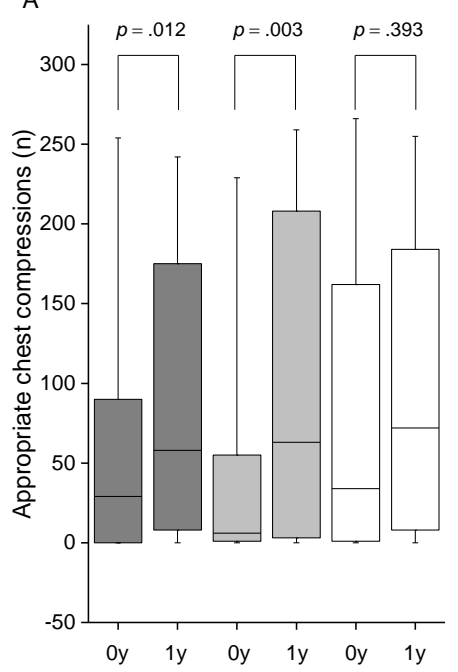

B

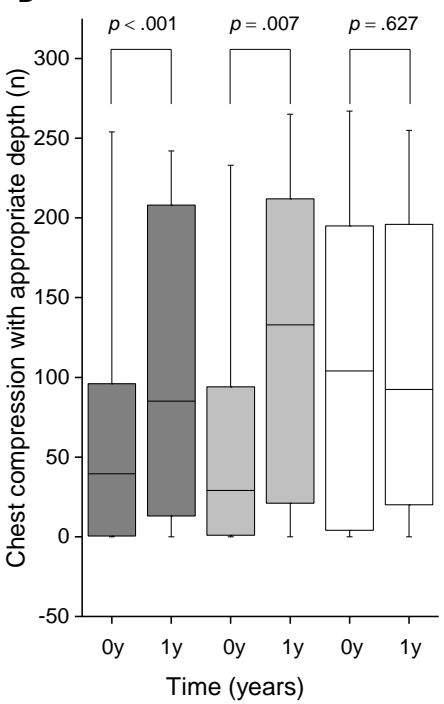

3-month group $(n=41)$

6-month group $(n=37)$

Control group $(n=32)$

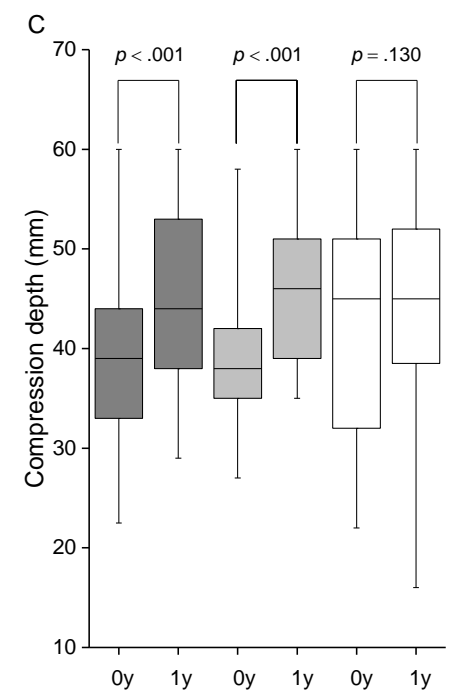

Figure 2. Box and whisker plot representing changes in chest compression skills over 1 year. Changes in chest compression skills within the group 1 year after a 45-minute chest compression-only cardiopulmonary resuscitation (CPR) training. (A) Number of appropriate chest compressions. (B) Number of chest compressions with appropriate depth. (C) Mean chest compression depth. OY, immediately after the chest compression-only CPR training; $1 \mathrm{Y}, 1$ year after the chest compression-only CPR training. Middle bar, median; box, interquartile range; whiskers, 2.5 th and 97.5 th percentiles

month (29 [0-90] vs $58[8-175], p=.012)$ and 6-month $(6[1-53]$ vs $63[3-208], p=.003)$ self-learning refresher training groups. The number of chest compressions with appropriate depth significantly differed 1 year and immediately after the training in the 3-month (40 [1-96] vs $85[13-208], p<.001)$ and 6-month (26 [1-94] vs 133 [21-212], $p=.007)$ groups. Chest compression depth at 1 year after the training was significantly deeper than that observed immediately after the training in the 3month (39 $\mathrm{mm}$ [33-44] vs $44 \mathrm{~mm}[38-53], p<.001)$ and 6-month (38 $\mathrm{mm}$ [35-42] vs $46 \mathrm{~mm}$ [39-51], $p<$ $.001)$ self-learning refresher training groups. In the control group, no significant differences were observed between the two time periods (at 1 year compared with immediately after the training) with regard to the CPR skills.

\section{Discussion}

International Journal of First Aid Education, Vol.2, Issue 2
Self-learning refresher training with short-term intervals did not contribute to retention of chest compressions with appropriate depth one year after the chest compression-only CPR training. However, analysis by paired comparison revealed that participants that underwent short intervals of self-retraining every 3 or 6 months after the chest compression-only CPR training maintained the number of appropriate chest compressions, the number of chest compressions with appropriate depth, and chest compression depth.

However, our results are not consistent with those reported in previous studies, suggesting the effectiveness of low-dose retraining for long-term retention (Nishiyama et al., 2015; Oermann et al., 2011). The discordant results may be due to three reasons. First, the two different types of short-interval selflearning groups in this study demonstrated a tendency for low numbers of chest compressions with appropriate depth immediately after the chest 
compression-only CPR training (compared to the previous study), although no significant differences were observed among the three groups. In a previous study, the number of chest compressions with appropriate depth immediately after the chest compression-only CPR training was $88.8 \pm 67.0$ (mean \pm standard deviation) (Nishiyama et al., 2009). Thus, in the current study, participants with poor CPR skills may have been allocated to two different short-interval selflearning groups. Although we adjusted for age, sex, previous CPR training, and the number of chest compressions with appropriate depth immediately after the chest compression-only CPR training using GLM, the results of the present study failed to yield statistical significance. Therefore, we suggest that future studies should consider a baseline of CPR skills as well as sex as allocation factors. Second, the practice of chest compressions using the Mr.PUSH PRO CPR501 device for self-learning may be insufficient to acquire competence in performing chest compressions. A previous study reported that a 1-min self-retraining could not achieve an adequate chest compression depth (Nishiyama et al., 2017). Based on this previous study, the present study was designed to allow participants to repeat chest compressions for a minimum of $1 \mathrm{~min}$ and a maximum of $5 \mathrm{~min}$, if they did not initially fulfill the criteria. However, it appears that two different shortinterval self-learning groups did not improve their CPR skills even after a year of chest compression-only CPR training because of insufficient repetition or the short training time of one min. Third, there may have been differences in the devices used during the chest compression-only CPR training, self-retraining, and the evaluation of chest compression, which may have affected the results.

As shown in Figure 2, our results concur with those reported in Sutton et al. (2011)'s study, suggesting that retraining of participants with poor skills likely contributes to improving the quality of chest compressions. Previous studies reported that low-dose CPR training could improve CPR skill performance (Nishiyama et al., 2017; Oermann et al., 2011; Sutton et al., 2011). The 2015 CoSTR recommended more frequent training to maintain CPR skills. Therefore, short self-learning refresher training described in this study may be useful in acquiring appropriate chest compression skills in participants with poor CPR skills. Moreover, in the control group, the number of appropriate chest compressions, the number of chest compressions with appropriate depth, and chest compression depth at 1 year after the training were similar compared with those observed immediately after the training. In a previous study, the compression-only CPR training program for the general public was shown to retain CPR skills compared with the conventional CPR training program (Nishiyama et al., 2014), and might help maintain their CPR skills. By chance, the control group showed a tendency to perform more chest compressions with appropriate depth immediately after the training among the three groups. Furthermore, $7(21.9 \%)$ participants in the control group arbitrarily participated in CPR training during the study period, and no significant differences were found among the 3 groups. These appear to be because the chest compression skills in the control group did not decline.

Analysis by GLM indicated that self-retraining at both 3-monthly and 6-monthly intervals was significantly associated with the total chest compressions one year after the chest compression-only CPR training. The paired comparison also demonstrated that participants that underwent self-retraining every three or six months after the chest compression-only CPR training maintained the number of appropriate chest compressions, the number of chest compressions with appropriate depth, and chest compression depth. Taken together, these results suggest that short self-learning refresher training every six months after the chest compression-only CPR training may help trainees sufficiently preserve chest compression skills. Smith, Gilcreast, and Pierce (2008) established that nurses who received basic life-support training retained their skills for 3-6 months, albeit with loss of skill performance. However, a previous study revealed that refresher training six months after the chest compression-only CPR training could help trainees retain chest compression skills compared with those that did not receive refresher training during the 1 -year period 
(Nishiyama et al., 2015). Moreover, a study reported the deterioration of CPR performance after six months of the first training regardless of age (Christenson et al., 2007). Therefore, it is reasonable to provide refresher CPR training programs six months after the chest compression-only CPR training.

\section{Limitations}

This study had several limitations. First, CPR performance in actual settings where CPR-trained bystanders could easily panic remains unclear, since we assessed the participants' CPR skills using a simulation method. Second, participants were all university students, whose median age was 20 years, and with higher educational experiences. Thus, further studies are required to evaluate the effectiveness of short selflearning refresher training in other age groups as well as different education backgrounds. Third, the effect of the baseline skills may have influenced the results of this study. Although there were no significant differences in the baseline characteristics among the three groups, the control group tended to perform chest compressions with appropriate depth after the initial CPR compression training. Hence, we should have included baseline CPR skills as an allocation factor.

\section{Conclusion}

In conclusion, a short self-learning refresher training every three or six months after the chest compressiononly CPR training could not be attributed to improvement in long-term retention of chest compressions with appropriate depth in university students. However, short, frequent self-retraining in persons with poor skills might be useful in acquiring and retaining chest compression skills. Hence, further studies are needed to clarify the optimal interval and methods for CPR retraining.

\section{References}

Andresen, D., Arntz, H. R., Gräfling, W., Hoffmann, S., Hofmann, D., Kraemer, R., . . Wegscheider, K. (2008). Public access resuscitation program including defibrillator training for laypersons: A randomized trial to evaluate the impact of training course duration. Resuscitation, 76, 419-424. doi:10.1016/j.resuscitation.2007.08.019

Ashton, A., McCluskey, A., Gwinnutt, C. L., \& Keenan, A. M. (2002). Effect of rescuer fatigue on performance of continuous external chest compressions over 3 min. Resuscitation, 55(2), 151-155. doi:10.1016/S03009572(02)00168-5

Bhanji, F., Finn, J. C., Lockey, A., Monsieurs, K., Frengley, R., Iwami, T., .. . Teams Chapter, C. (2015). Part 8: Education, Implementation, and Teams: 2015 International Consensus on Cardiopulmonary Resuscitation and Emergency Cardiovascular Care Science With Treatment Recommendations. Circulation, 132(16 Suppl 1), S242-268. doi:10.1161/CIR.0000000000000277

Christenson, J., Nafziger, S., Compton, S., Vijayaraghavan, K., Slater, B., Ledingham, R., . . McBurnie, M. A. (2007). The effect of time on CPR and automated external defibrillator skills in the Public Access Defibrillation Trial. Resuscitation, 74, 52-62. doi:10.1016/j.resuscitation.2006.11.005

Gyllenborg, T., Granfeldt, A., Lippert, F., Riddervold, I. S., \& Folke, F. (2017). Quality of bystander cardiopulmonary resuscitation during real-life out-of-hospital cardiac arrest. Resuscitation, 120, 63-70. doi:10.1016/j.resuscitation.2017.09.006

Japan Resuscitation Council. (2016). Japan Resuscitation Council Resuscitation Guidelines 2015. Tokyo: IgakuShoin Ltd.

Niles, D. E., Nishisaki, A., Sutton, R. M., Elci, O. U., Meaney, P. A., O'Connor, K. A., . . Nadkarni, V. (2017). 
Improved Retention of Chest Compression Psychomotor Skills With Brief "Rolling Refresher" Training. Simulation in Healthcare, 12, 213-219. doi:10.1097/sih.0000000000000228

Nishiyama, C., Iwami, T., Kawamura, T., Ando, M., Kajino, K., Yonemoto, N., . . Nonogi, H. (2009). Effectiveness of simplified chest compression-only CPR training program with or without preparatory self-learning video: a randomized controlled trial. Resuscitation, 80(10), 1164-1168. doi:10.1016/j.resuscitation.2009.06.019

Nishiyama, C., Iwami, T., Kitamura, T., Ando, M., Sakamoto, T., Marukawa, S., \& Kawamura, T. (2014). Long-term retention of cardiopulmonary resuscitation skills after shortened chest compression-only training and conventional training: A randomized controlled trial. Academic Emergency Medicine, 21, 47-54. doi:10.1111/acem.12293

Nishiyama, C., Iwami, T., Murakami, Y., Kitamura, T., Okamoto, Y., Marukawa, S., . . Kawamura, T. (2015). Effectiveness of simplified 15-min refresher BLS training program: A randomized controlled trial. Resuscitation, 90, 56-60. doi:10.1016/j.resuscitation.2015.02.015

Nishiyama, C., Shimamoto, T., Kiyohara, K., Kawamura, T., Kitamura, T., Sakamoto, T., \& Iwami, T. (2017). Effectiveness of a one-minute self-retraining for chest compression-only cardiopulmonary resuscitation: Randomized controlled trial. AEM Education and Training, 1(3), 200-207. doi:10.1002/aet2.10034

Nyman, J., \& Sihvonen, M. (2000). Cardiopulmonary resuscitation skills in nurses and nursing students. Resuscitation, 47, 179-184. doi:10.1016/S0300-9572(00)00226-4

Oermann, M. H., Kardong-Edgren, S. E., \& Odom-Maryon, T. (2011). Effects of monthly practice on nursing students' CPR psychomotor skill performance. Resuscitation, 82, 447-453. doi:10.1016/j.resuscitation.2010.11.022

Smith, K. K., Gilcreast, D., \& Pierce, K. (2008). Evaluation of staff's retention of ACLS and BLS skills. Resuscitation, 78, 59-65. doi:10.1016/j.resuscitation.2008.02.007

Sutton, R. M., Niles, D., Meaney, P. A., Aplenc, R., French, B., Abella, B. S., . . Nadkarni, V. (2011). Low-dose, high-frequency CPR training improves skill retention of in-hospital pediatric providers. Pediatrics, 128 , e145-151. doi:10.1542/peds.2010-2105

Wik, L., Brennan, R. T., \& Braslow, A. (1995). A peer-training model for instruction of basic cardiac life support. Resuscitation, 29(2), 119-128. doi:10.1016/0300-9572(94)00835-4 